TRANSACTIONS OF THE

AMERICAN MATHEMATICAL SOCIETY

Volume 356, Number 11, Pages 4273-4285

S 0002-9947(04)03613-X

Article electronically published on May 28, 2004

\title{
LOCALIZATION FOR A POROUS MEDIUM TYPE EQUATION IN HIGH DIMENSIONS
}

\author{
CHANGFENG GUI AND XIAOSONG KANG
}

\begin{abstract}
We prove the strict localization for a porous medium type equation with a source term, $u_{t}=\nabla\left(u^{\sigma} \nabla u\right)+u^{\beta}, x \in \mathbf{R}^{N}, N>1, \beta>\sigma+1, \sigma>0$, in the case of arbitrary compactly supported initial functions $u_{0}$. We also otain an estimate of the size of the localization in terms of the support of the initial data $\operatorname{supp} u_{0}$ and the blow-up time $T$. Our results extend the well-known one dimensional result of Galaktionov and solve an open question regarding high dimensions.
\end{abstract}

\section{INTRODUCTION}

In this paper we consider the Cauchy problem for a porous medium equation with a source term

$$
\left\{\begin{array}{l}
u_{t}=\nabla\left(u^{\sigma} \nabla u\right)+u^{\beta} \text { in } \mathbf{R}^{N} \times[0, T), \\
u(x, 0)=u_{0}(x) \text { in } \mathbf{R}^{N}
\end{array}\right.
$$

where $\beta>\sigma+1, \sigma>0$ are constants, $N>1$. This equation has been widely used as a simple model for a nonlinear heat propagation in a reactive medium, where $u$ denotes temperature.

We assume that $u_{0}$ is a nonnegative not identically zero funciton. By a solution of (1.1) we mean a nonnegative continuous function $u(x, t)$ with $u^{\sigma+1} \in$ $C\left([0, T), H_{l o c}^{1}\left(\mathbf{R}^{N}\right)\right)$, which also satisfies (1.1) in the following weak sense:

$$
\int_{0}^{T} \int_{\mathbf{R}^{N}}\left[u \phi_{t}-u^{\sigma} \nabla u \cdot \nabla \phi+u^{\beta} \phi\right] d x d t+\int_{\mathbf{R}^{N}} u_{0}(x) \phi(x, 0) d x=0
$$

for all $\phi \in C_{0}^{\infty}\left(\mathbf{R}^{N} \times[0, T)\right)$.

Under the above assumptions, local existence in time, uniqueness and pointwise comparison principle for solutions can be proven for (1.1). Indeed, the solution $u(x, t)$ is a classical solution at any point where $u>0$; see for instance [14]. It is well known [16] that when $\sigma>0,1<\beta \leq \sigma+1+\frac{2}{N}$, the solution of (1.1) always blows up in finite time, while for $\beta>\sigma+1+\frac{2}{N}$ the blow-up occurs if $u_{0}$ is large enough. In the latter case there also exist small solutions which are global in time. By finite blow-up we mean there is a $T>0$ such that $\|u(\cdot, t)\|_{\infty}$ is finite for all $t \in[0, T)$, but

$$
\limsup _{t \rightarrow T^{-}}\|u(\cdot, t)\|_{\infty}=+\infty
$$

Received by the editors September 18, 2002.

2000 Mathematics Subject Classification. Primary 35K15, 35K55, 35K65; Secondary 35J40.

Key words and phrases. Porous medium type equation with source, localization property, blow-up, self-similar solutions, comparison. 
In our study we shall restrict attention to unbounded blow-up solutions to (1.1) with $\beta>\sigma+1$.

First we recall some known facts briefly; more details can be found in surveys [15], [7], or [16]. Like the standard porous medium equation $u_{t}=\nabla\left(u^{\sigma} \nabla u\right)$, equation (1.1) describes a process with a finite speed of propagation of disturbances, i.e., if the initial data $u_{0}$ are compactly supported, then for all $t \in(0, T)$,

$$
\operatorname{supp} u(\cdot, t)=\left\{x \in \mathbf{R}^{N} \mid u(x, t)>0\right\}
$$

is a bounded set. The finite speed propagation phenomenon is due to the degeneracy of the equation at the interface $u=0$, and is one of the most important facts about porous medium type equations which makes these equations very interesting objects of study.

Some definitions are in order. An unbounded solution of problem (1.1) is called strictly localized if

$$
\Omega_{L}\left(u_{0}\right)=\left\{x \in \mathbf{R}^{N} \mid u\left(x, T^{-}\right)=\limsup _{t \rightarrow T^{-}} u(x, t)>0\right\}
$$

is bounded, effectively localized if the blow-up set $B U\left(u_{0}\right)$ is bounded, where

$$
B U\left(u_{0}\right)=\left\{x \in \mathbf{R}^{N} \mid \text { there exist } t_{n} \rightarrow T^{-}, x_{n} \rightarrow x \text { with } u\left(x_{n}, t_{n}\right) \rightarrow+\infty\right\} .
$$

Observe that by definition of $T, B U\left(u_{0}\right)$ is a nonempty closed set.

Similar to the porous medium equation [17], the solution $u$ to (1.1) enjoys the retention property $\operatorname{supp} u\left(\cdot, t_{1}\right) \subseteq \operatorname{supp} u\left(\cdot, t_{2}\right)$ for every $0 \leq t_{1}<t_{2}<T$. This can be proven by the barrier method. Hence strict localization happens only if $u_{0}$ has a compact support.

For $1<\beta<\sigma+1$, blow-up could happen in the whole space $\mathbf{R}^{N}$ even when the initial data are compactly supported. Hence there is no localization in this case. For $\beta=\sigma+1$, the blow-up set $B U\left(u_{0}\right)$ can only be a bounded region, so it is called regional blow-up. In this case, one can also establish the symmetry of the blow-up set and describe the blow-up profile precisely (see [11, [3]). However, we expect that the situation $\beta>\sigma+1$ is different and should be similar to the semilinear case where $\sigma=0, \beta>1$. In the latter case, it has been shown [18] that the blow-up set has locally finite $(N-1)$-Hausdorff measure. In particular its Lebesgue measure is zero. In some special cases, such as the case when $u_{0}$ is radially decreasing, even single-point blow-up can be proven [20, 4] for the semilinear case. Note that for semilinear heat equations, the asymptotic blow-up profile is quite well understood [10].

In one spatial dimension case, for $\sigma>0$ and $\beta>\sigma+1$, Galaktionov has demonstrated in [5] the strict localization for intial data with compact support, hence the blow-up is at most regional. His argument is based on some Sturmian type theorems (intersection comparison technique or lap numbers), which are essentially of ODE nature. The approach may be extended to deal with radially symmetric solutions in arbitrary dimensions.

However, in higher dimensions the problem of spectrum of nonsymmetric blowup remains open, as emphasized in [16]. Little seems known regarding either strict or effective localization, or any kind of Hausdorff measure estimate. Though the intersection comparison technique has shown to be a strong tool in analyzing nonlinear diffusions in one space variable, it seems hard to extend it to higher dimensions unless an assumption like radial symmetry is made. 
In this paper we will show the strict localization in higher dimensions for arbitrary compactly supported initial data, without any symmetry condition. We also obtain an estimate for the size of the support, hence it provides an affirmative answer to an open problem in [16] (Question 5, p. 315). Here is our main result.

Theorem 1.1. Suppose that $u$ is the solution to the problem (1.1) with compactly supported initial $u_{0}$ and that $T$ is the blow-up time, where $\beta>\sigma+1$. Then there exists a $K>0$, depending on $\sigma, \beta, N, u_{0}$ and $T$, such that for all $t \in[0, T)$,

$$
\operatorname{supp} u(\cdot, t) \subset B(0, K) .
$$

We also investigate the question of effective localization. In particular, we obtain results on the case where initial $u_{0}$ is not necessarily of compact support but satisfies some extra symmetry and decay conditions. The following theorem provides some insight on another open problem in [16].

Theorem 1.2. Suppose $u_{0}(x)=u_{0}(r)$, where $r=|x|$, and $u_{0 r} \leq 0$. Suppose in addition that $u_{0}<C|x|^{-\frac{2}{\beta-(\sigma+1)}}$, as $|x| \rightarrow \infty$, where $0<C<C_{s}$. Then u will decay to 0 uniformly in all $t \in(0, T)$. Hence in this case the effective localization holds.

Note that $C_{s}$ is a positive constant which will be defined in section 2 .

Our approach is inspired by [3], where among other things the localization for $\beta=$ $\sigma+1$ is proved. However the case $\beta>\sigma+1$ is quite different here. The difficulty is, when $\beta>\sigma+1$, there are no compactly supported self-similar solutions. There are only some small self-similar supersolutions with compact support in more restrictive $\sigma, \beta$ ranges. It seems difficult to compare these self-similar (super)solutions with our solutions directly. Our strategy is to establish first the uniform bound for $u$ when $|x|$ is large, and then try to compare it with the self-similar solution for $\beta=\sigma+1$ there, using the differential inequality for our solutions at infinity. Another difference is that our similarity transformed operator is not translation invariant, hence one must use the original solution $u$ as well as the transformed one (which is globally defined in $t$ ) simultaneously in order to achieve different estimates. In fact, we will apply comparison technique on the original solution to get pointwise estimate, while use a monotonicity argument to obtain a bound estimate on the transformed solution.

We begin with a section devoted to self-similar representation and self-similar solutions; these results will be used in section 3 where Theorem 1.1 is proven. Finally we sketch the proof of Theorem 1.2 and discuss some related issues.

\section{SELF-SIMILAR REPRESENTATIONS AND SELF-SIMILAR SOLUTIONS}

Let us introduce the similarity representation of $u$, the solution to (1.1)

$$
v(\xi, \tau)=(T-t)^{\frac{1}{\beta-1}} u(x, t),
$$

where $\xi=\frac{x}{(T-t)^{m}} \in \mathbf{R}^{N}, \tau=-\ln \frac{T-t}{T} \in[0, \infty), m=\frac{\beta-(\sigma+1)}{2(\beta-1)} \geq 0$ for $\beta \geq \sigma+1$. Note that we have $\xi=T^{-m} e^{\tau m} x, T-t=T e^{-\tau}$.

Direct calculation leads to the following Cauchy problem for $v$ :

$$
\left\{\begin{array}{l}
v_{\tau}=\nabla\left(v^{\sigma} \nabla v\right)-m \nabla v \cdot \xi-\frac{v}{\beta-1}+v^{\beta} \text { in } \mathbf{R}^{N} \times(0, \infty) \\
v(\xi, 0)=T^{\frac{1}{\beta-1}} u_{0}\left(\xi T^{m}\right) \text { in } \mathbf{R}^{N} .
\end{array}\right.
$$

Notice that if $\operatorname{supp} u_{0} \subset B\left(0, R_{0}\right)$, then $\operatorname{supp} v(\cdot, 0) \subset B\left(0, R_{0} T^{-m}\right)$, and vice versa. As usual $B(0, R)$ means the ball in $\mathbf{R}^{N}$ centered at the origin with radius $R$. 
If $v$ is $\tau$ independent, i.e., the stationary solution to (2.2), then $u$ is called a self-similar solution. Let $u(x, t)=(T-t)^{-\frac{1}{\beta-1}} \theta(\xi)$, where $\xi=\frac{x}{(T-t)^{m}}$; then $\theta$ satisfies

$$
\nabla\left(\theta^{\sigma} \nabla \theta\right)-\frac{\theta}{\beta-1}-m \nabla \theta \cdot \xi+\theta^{\beta}=0
$$

Note that asymptotic blow-up properties of the solutions are usually governed by the nontrivial self-similar solutions, and self-similar solutions with the same blowup times act as "attractors" for the blow-up solutions in some sense; see Chapter IV in [16] and an extended list of references therein.

Next we just collect some facts without proofs about the one dimesional selfsimilar solutions and their properties. For details please see [16]. Notice that we will use notations different from [16].

Proposition 2.1 ([16], p. 195). For $\beta>\sigma+1, N=1$, equation (2.3) has a positive even solution $\theta_{s}=\theta_{s}\left(\xi_{1}\right), \xi_{1} \in \mathbf{R}^{1}$, which is strictly decreasing and satisfies

$$
\left\{\begin{array}{l}
\left(\theta_{s}^{\sigma} \theta_{s}^{\prime}\right)^{\prime}-m \theta_{s}^{\prime} \xi_{1}-\frac{\theta_{s}}{\beta-1}+\theta_{s}^{\beta}=0 \\
\theta_{s}(0)=\theta_{0}>\theta_{H} \equiv\left(\frac{1}{\beta-1}\right)^{\frac{1}{\beta-1}} \\
\theta_{s}^{\prime}(0)=0, \theta_{s} \rightarrow 0 \text { as } \xi_{1} \rightarrow \infty
\end{array}\right.
$$

Here $\theta_{H}$ is the spatially homogeneous solution of equation (2.3). The fact $\theta_{0}>$ $\theta_{H}$ will play some role in later proofs. The proof of this proposition is based on a shooting type argument. If we define $u_{s}\left(x_{1}, t\right) \equiv(T-t)^{-\frac{1}{\beta-1}} \theta_{s}\left(\xi_{1}\right)$, it solves

$$
\left\{\begin{array}{l}
\left(u_{s}\right)_{t}=\left(u_{s}^{\sigma} u_{s}^{\prime}\right)^{\prime}+u_{s}^{\beta}, \\
u_{s}(0, t)=(T-t)^{-\frac{1}{\beta-1}} \theta_{0},
\end{array}\right.
$$

where $\xi_{1}=\frac{x_{1}}{(T-t)^{m}}, m=\frac{\beta-(\sigma+1)}{2(\beta-1)}$.

In addition $\theta_{s}$ has the following asymptotics ([16], p. 190):

$$
\theta_{s}\left(\xi_{1}\right)=C_{s} \xi_{1}^{-\frac{2}{\beta-(\sigma+1)}}\left(1+\nu\left(\xi_{1}\right)\right), \nu\left(\xi_{1}\right) \rightarrow 0 \text { as } \xi_{1} \rightarrow+\infty,
$$

where $C_{s}=C_{s}(\sigma, \beta)>0$ is a constant. As $x_{1} \rightarrow \infty$, we have $\frac{x_{1}}{(T-t)^{m}}=\xi_{1} \rightarrow \infty$ uniformly for $t \in(0, T)$. So one may expect

$$
u_{s}\left(x_{1}, t\right) \rightarrow C_{s}(T-t)^{-\frac{1}{\beta-1}}\left|\frac{x_{1}}{(T-t)^{m}}\right|^{-\frac{2}{\beta-(\sigma+1)}}=C_{s}\left|x_{1}\right|^{-\frac{2}{\beta-(\sigma+1)}}
$$

uniformly for $t \in(0, T)$ as $x_{1} \rightarrow \infty$. Then one may conclude that $u_{s}$ uniformly (in $t$ ) decays to zero as $x_{1}$ goes to infinity. This argument can indeed be made rigorous due to a a surprising monotonicity property of self-similar solutions, namely $\frac{\partial u_{s}}{\partial t}>0$ for all $t \in(0, T)$. Therefore,

$$
u_{s}\left(x_{1}, t\right)<u_{s}\left(x_{1}, T^{-}\right) \equiv C_{s}\left|x_{1}\right|^{-\frac{2}{\beta-(\sigma+1)}}, x_{1} \in \mathbf{R}^{1}, x_{1} \neq 0,
$$

which implies

$$
\sup _{0<t<T} u_{s}\left(x_{1}, t\right) \rightarrow 0, \text { as }\left|x_{1}\right| \rightarrow \infty \text {. }
$$

In the literature this property is called "criticality"; one may find a proof for it in [16], pp. 197-198.

This fact will help us get the desired uniform decay for our solution later. 
Proposition 2.2 ([16], p. 180$)$. Let

$$
\vartheta\left(x_{1}\right)= \begin{cases}\left(\frac{2(\sigma+1)}{\sigma(\sigma+2)} \cos ^{2} \frac{\pi x_{1}}{L_{s}}\right)^{\frac{1}{\sigma}} & \text { if }\left|x_{1}\right|<\frac{L_{s}}{2}, \\ 0 & \text { if }\left|x_{1}\right| \geq \frac{L_{s}}{2},\end{cases}
$$

where $L_{s}=\frac{2 \pi}{\sigma}(\sigma+1)^{\frac{1}{2}}$. Then $\vartheta$ satisfies the following ODE:

$$
\left(\vartheta^{\sigma} \vartheta^{\prime}\right)^{\prime}-\frac{1}{\sigma} \vartheta+\vartheta^{\sigma+1}=0 .
$$

Furthermore, the function

$$
u_{1}\left(x_{1}, t\right)=(T-t)^{-\frac{1}{\sigma}} \vartheta\left(x_{1}\right)
$$

satisfies

$$
\left(u_{1}\right)_{t}=\left(\left(u_{1}\right)^{\sigma}\left(u_{1}\right)^{\prime}\right)^{\prime}+\left(u_{1}\right)^{\sigma+1} \text { in } \mathbf{R}^{1} \times(0, T) .
$$

The important thing here is that $u_{1}$ has compact support; this will help us establish the desired localization property.

Proposition 2.3 ([16], p. 219). For $\beta>\sigma+3$, there exist some small positive numbers $A$, a such that the globally defined (in time) function

$$
u_{2}\left(x_{1}, t\right)=(1+t)^{-\frac{1}{\beta-1}} \psi\left(\xi_{1}\right)
$$

satisfies

$$
\left(u_{2}\right)_{t} \geq\left(u_{2}^{\sigma}\left(u_{2}\right)^{\prime}\right)^{\prime}+u_{2}^{\beta}, \quad t>0,
$$

where $\xi_{1}=\frac{x_{1}}{(1+t)^{m}}, m=\frac{\beta-(\sigma+1)}{2(\beta-1)}, \psi\left(\xi_{1}\right)=A\left(1-\frac{\xi_{1}^{2}}{a^{2}}\right)_{+}^{\frac{1}{\sigma}}$.

As mentioned before, the global $N$-dimensional solutions exist only if $\beta>\sigma+$ $1+\frac{2}{N}$, so $\sigma+3$ is the optimal lower bound for $\beta$ to guarantee the existence of a global supersolution when $N=1$.

Similar to $u_{1}$, the support of $u_{2}$ is compact, but unlike $u_{1}$, its support may be time dependent and $\operatorname{supp} u_{2}(\cdot, t) \in B\left(0, a(T+1)^{m}\right)$ for all $t \in(0, T)$.

We will use this supersolution to establish a better estimate on the size of the support, when $\beta>\sigma+3$.

Among the above three functions $u_{s}, u_{1}, u_{2}$, either has the same blow-up time $T$ as $u$ does, or it is globally defined in time. So we can compare our solutions with these functions. In the following, without loss of generality we may assume that $u$ is a classical solution. In the case of degeneracy one may approximate the weak solution $u(x, t)$ by a sequence of classical positive solutions $\left\{u_{n}(x, t)\right\}$ satisfying uniformly parabolic problems, then pass to the limit.

\section{Proof of Theorem 1.1}

In this section we always assume that $u_{0}$ is compactly supported, $\operatorname{supp} u_{0} \subset$ $B\left(0, R_{0}\right), m=\frac{\beta-(\sigma+1)}{2(\beta-1)}>0$.

Several lemmas are in order. The first is essentially contained in [1. The proof is based on Alexandroff's reflection principle and comparison; we omit its elegant but elementary proof.

Lemma 3.1. Assume that $\operatorname{supp} u_{0} \subset B\left(0, R_{0}\right)$. Then for all $R>0$, one has

$$
\sup _{x \in \partial B\left(0, R+2 R_{0}\right)} u(x, t) \leq \inf _{x \in B(0, R)} u(x, t) \text { for all } t \in(0, T),
$$


i.e.,

$$
\sup _{\xi \in \partial B\left(0,\left(R+2 R_{0}\right) T^{-m} e^{\tau m}\right)} v(\xi, \tau) \leq \inf _{\xi \in B\left(0, R T^{-m} e^{\tau m}\right)} v(\xi, \tau), \text { for all } \tau>0 .
$$

In particular, since $m>0$,

$$
\sup _{\xi \in \partial B\left(0,\left(R+2 R_{0}\right) T^{-m} e^{\tau m}\right)} v(\xi, \tau) \leq \inf _{\xi \in B\left(0, R T^{-m}\right)} v(\xi, \tau), \text { for all } \tau>0 .
$$

The next lemma tells us that, roughly speaking, uniform boundedness yields uniform decay.

Lemma 3.2. Assume that there exists $R_{1}>0$ such that $v(\xi, 0)=0$ for $\left|\xi T^{m}\right| \geq R_{1}$, and $v(\xi, \tau)<\theta_{0}$ whenever $\left|\xi T^{m} e^{-\tau m}\right| \geq R_{1}$ for all $\tau>0$. Then

$$
\sup _{0<t<T} u(x, t) \rightarrow 0, \text { as }|x| \rightarrow \infty .
$$

Proof. By the definition of $v$, we know that the fact

$$
v(\xi, 0)=0 \text { for }\left|\xi T^{m}\right| \geq R_{1}
$$

is equivalent to

$$
u_{0}(x)=0 \text { for }|x| \geq R_{1},
$$

and the inequality

$$
v(\xi, \tau)<\theta_{0} \text { for }\left|\xi T^{m} e^{-\tau m}\right| \geq R_{1}, \tau>0
$$

is equivalent to

$$
u(x, t)<\theta_{0}(T-t)^{-\frac{1}{\beta-1}} \text { for }|x| \geq R_{1}, t>0 .
$$

Set $w(x, t) \equiv u_{s}\left(x_{1}-R_{1}, t\right)$, where $x=\left(x_{1}, x^{\prime}\right) \in \mathbf{R}^{N}, x_{1} \in \mathbf{R}^{1}$ ( $u_{s}$ is defined in section 2 under Proposition 2.1).

So by the assumption we obtain

$$
\left\{\begin{array}{l}
w_{t}=\nabla\left(w^{\sigma} \nabla w\right)+w^{\beta} \text { in }\left\{x \in \mathbf{R}^{N} \mid x_{1} \geq R_{1}\right\} \times[0, T) \\
w(x, 0) \geq u(x, 0) \equiv 0 \text { if } x_{1} \geq R_{1} \\
w(x, t)=\theta_{0}(T-t)^{-\frac{1}{\beta-1}}>u(x, t) \text { on }\left\{x_{1}=R_{1}\right\} \times[0, T) .
\end{array}\right.
$$

By comparison, it follows that

$$
u(x, t) \leq w(x, t)=u_{s}\left(x_{1}-R_{1}, t\right)
$$

for all $x_{1} \geq R_{1}, 0<t<T$.

According to (2.5),

$$
u(x, t) \leq C_{s}\left|x_{1}-R_{1}\right|^{-\frac{2}{\beta-(\sigma+1)}}
$$

as long as $x_{1}>R_{1}, t \in[0, T)$.

Hence

$$
\sup _{0<t<T} u(x, t) \rightarrow 0 \text { as } x_{1} \rightarrow+\infty .
$$

Since this argument can be repeated in every direction, the lemma is proved.

Lemma 3.3. Suppose $\beta>\sigma+1$, supp $u_{0} \subset B\left(0, R_{0}\right)$, and $\sup _{0<t<T} u(x, t) \rightarrow$ 0 as $|x| \rightarrow \infty$. Then there exists a $K>0$ such that

$$
\operatorname{supp} u(\cdot, t) \subset B(0, K)
$$

for all $t \in[0, T)$. 
Proof. Recall from Proposition 2.2 that

$$
u_{1}\left(x_{1}, t\right)=(T-t)^{-\frac{1}{\sigma}} \vartheta\left(x_{1}\right)
$$

satisfies

$$
\left(u_{1}\right)_{t}=\left(\left(u_{1}\right)^{\sigma}\left(u_{1}\right)^{\prime}\right)^{\prime}+\left(u_{1}\right)^{\sigma+1} \text { in } \mathbf{R}^{1} \times(0, T)
$$

and

$$
\operatorname{supp} u_{1}(\cdot, t) \subset\left\{x_{1} \in \mathbf{R}^{1}|| x_{1} \mid \leq \frac{L_{s}}{2}\right\} .
$$

By assumption, there exists $R_{2} \geq R_{0}$ such that

$$
u(x, t)<\min \left\{T^{-\frac{1}{\sigma}} \vartheta(0), 1\right\} \text { for all }|x| \geq R_{2}, t \in[0, T) .
$$

Consider

$$
w_{1}(x, t) \equiv u_{1}\left(x_{1}-R_{2}, t\right), x \in \mathbf{R}^{N} .
$$

Then we have

$$
\left(w_{1}\right)_{t}=\nabla\left(\left(w_{1}\right)^{\sigma} \nabla w_{1}\right)+\left(w_{1}\right)^{\sigma+1} \text { in }\left\{x \in \mathbf{R}^{N} \mid x_{1} \geq R_{2}\right\} \times(0, T)
$$

while

$$
u_{t}=\nabla\left(u^{\sigma} \nabla u\right)+u^{\beta} \leq \nabla\left(u^{\sigma} \nabla u\right)+u^{\sigma+1}
$$

in $\left\{x \mid x_{1} \geq R_{2}\right\} \times[0, T)$. On the parabolic boundary, one has

$$
w_{1}(x, 0) \geq u(x, 0) \equiv 0 \text { in }\left\{x \mid x_{1}>R_{2}\right\},
$$

and

$$
w_{1}(x, t)=(T-t)^{-\frac{1}{\sigma}} \vartheta(0) \geq T^{-\frac{1}{\sigma}} \vartheta(0)>u(x, t) \text { in }\left\{x \mid x_{1}=R_{2}\right\} \times[0, T) .
$$

By comparison, $w_{1}(x, t) \geq u(x, t)$ in $\left\{x \mid x_{1} \geq R_{2}\right\} \times[0, T)$.

Hence

$$
\operatorname{supp} u(\cdot, t) \subset\left\{x \mid x_{1} \leq R_{2}+\frac{L_{s}}{2}\right\}
$$

Repeating this argument in every direction, we know that $\operatorname{supp} u(\cdot, t) \subset B(0, K)$, where $K=R_{2}+\frac{L_{s}}{2}$.

Hence if we can prove that the hypothesis of Lemma 3.2 is satisfied, Theorem 1.1 will be a simple consequence of the above two lemmas.

Before going through the whole proof of the theorem, we would like to estimate the support size, i.e., the value of $K$ in Lemma 3.3.

Let us accept the hypothesis in Lemma 3.2 for a moment (we will see later that the proof of this fact does not depend on Lemma 3.2 and Lemma 3.3). From the proof of Lemma 3.2 one knows

$$
u(x, t) \leq C x_{i}^{-\frac{2}{\beta-(\sigma+1)}}
$$

for $x_{i}>R, i=1, \ldots, N$, where $R>R_{1}, C>0$ are constants, and $C$ is independent of $T$. For example, one can choose $R=k R_{1}, C=2 C_{s}$ for some $k=k(\sigma, \beta)>0$.

In Lemma $3.3 R_{2}$ is chosen to satisfy

$$
u(x, t)<C T^{-\frac{1}{\sigma}} \text { when }|x|>R_{2} .
$$

Notice that here and in what follows, $C$ represents a generic constant that depends on $\sigma, \beta, N$ only. It may change from line to line.

Hence we can choose $R_{2}>R$ so that

$$
R_{2}^{-\frac{2}{\beta-(\sigma+1)}} \leq C T^{-\frac{1}{\sigma}} \text {. }
$$


Later on, we will see that $R_{1}$ could be chosen as $R_{1}=2 R_{0}+C T^{m}$, where $C$ is independent of $R_{0}, T$, thus $R_{2}$ is the form

$$
R_{2}=C_{0}+C_{1} T^{m}+C_{2} T^{\frac{\beta-(\sigma+1)}{2 \sigma}},
$$

where $C_{i}$ is independent of $T, i=0,1,2$, and only $C_{0}$ depends on $R_{0}$.

Now we are going to prove the theorem. In addition to the differential inequalities and the comparison principle, we will use the monotonicity of the solution to an auxiliary equation.

Proof of Theorem 1.1. Lemmas 3.2 and 3.3 reduce the proof to finding a number $R_{1} \geq R_{0}$ such that $v(\xi, \tau)<\theta_{0}$ whenever $\left|\xi T^{m} e^{-\tau m}\right| \geq R_{1}$ for all $\tau>0$.

To this end we consider, for fixed numbers $R>0, Q>0, t_{0} \geq 0$, the initialboundary value problem

$$
\left\{\begin{array}{l}
p_{t}=\nabla\left(p^{\sigma} \nabla p\right)-m \nabla p \cdot \xi-\frac{p}{\beta-1}+p^{\beta} \text { in } B(0, R) \times\left(t_{0}, \infty\right) \\
p(x, t)=0 \text { on } \partial B(0, R) \times\left[t_{0},+\infty\right) \\
p\left(x, t_{0}\right)=Q \text { on } B(0, R)
\end{array}\right.
$$

Let $\lambda_{R}$ denote the first eigenvalue of the Laplacian under Dirichlet condition on $B(0, R)$. Then $\lambda_{R}=\frac{\lambda_{1}}{R^{2}}$. We make the following claim.

Claim. If $R^{2}>\lambda_{1}$ and

$$
Q>Q^{*}(R) \equiv\left[\frac{\sigma+1}{\sigma+1-\lambda_{R}}\left(\frac{\lambda_{R}}{\sigma+1}+\frac{1}{\beta-1}\right)\right]^{\frac{1}{\beta-1}},
$$

then the solution of problem (3.2) blows up in finite time.

Let us accept for the moment the validity of this claim and conclude the proof of the theorem. Set $R_{1}=2 R_{0}+R T^{m}$ where $R$ is to be chosen later. We claim that

$$
\sup _{\xi \in \partial B\left(0, R_{1} T^{-m} e^{\tau m}\right)} v(\xi, \tau) \leq Q^{*}(R)
$$

for all $\tau>0$.

Otherwise, by (3.1) in Lemma 3.1 there exists $t_{0}>0$ such that $\inf _{\xi \in B(0, R)} v\left(\xi, t_{0}\right)$ $>Q^{*}(R)$.

Then $v$ is a supersolution of (3.2) for this $t_{0}$ and some $Q>Q^{*}(R)$. Since the solution to (3.2) blows up in finite time by the claim, so does $v$. This is a contradiction which proves (3.3).

By Proposition 2.1 we have $Q^{*}(R) \rightarrow \theta_{H} \equiv\left(\frac{1}{\beta-1}\right)^{\frac{1}{\beta-1}}<\theta_{0}$. Then it is easy to find an $R=R(\sigma, \beta, N)>0$ so large that for all $R>R(\sigma, \beta, N)$ we have $\theta_{0}>Q^{*}(R)$. Therefore the theorem is implied by (3.3), Lemmas 3.2 and 3.3.

It remains to prove the claim. The key ingredients are the symmetry of $p$ and its monotonicity.

Observe that for all $A \in O(N)$, the orthonormal group in $R^{N}, p(A \cdot x, t)$ is also a solution to (3.2). By uniqueness, $p(A \cdot x, t) \equiv p(x, t)$, for all $t>t_{0}$. Hence $p(x, t)=p(r, t)$, where $r=|x|$. So $p_{r}(0, t)=0$ for all $t$.

Now the problem is reduced to the one space-variable case, for which we can use a trick in [4.

Set $z(r, t)=r^{N-1} p_{r}(r, t),(r, t) \in[0, R) \times\left[t_{0}, \infty\right)$.

One has

$$
z\left(r, t_{0}\right)=0, \text { for } r \in(0, R)
$$


and

$$
z(0, t)=0 \text { for all } t \in\left[t_{0},+\infty\right) .
$$

Since $p>0$ in $B(0, R) \times\left[t_{0},+\infty\right), p=0$ on $\partial B(0, R) \times\left[t_{0},+\infty\right)$, the strong maximum principle yields that $z<0$ for all $t>t_{0}$ when $r=R$.

In addition $z$ satisfies the following linear PDE:

$$
z_{t}=\left(p^{\sigma} z\right)_{r r}-\frac{N-1}{r}\left(p^{\sigma} z\right)_{r}+\left(\beta p^{\beta-1}+m(N-2)-\frac{1}{\beta-1}\right) z-m r z_{r},
$$

in $(0, R) \times\left(t_{0}, \infty\right)$.

Notice that in the PDE above, the coefficient of $z$ is bounded on $(0, R) \times\left[t_{0}, t_{1}\right)$, as long as $t_{1}$ is smaller than the existing time of $p$. So we can apply the maximum principle for (3.4) to conclude $z \leq 0$. Hence $p_{r}(r, t) \leq 0$ for all $r<R, t>t_{0}$ as long as $p$ does not blow up.

Now set

$$
S(t)=\int_{B(0, R)} p(x, t) \phi(x) d x,
$$

where $R^{2}>\lambda_{1}, \phi$ is the first Dirichlet eigenfunction of the Laplacian, normalized so that $\int_{B(0, R)} \phi=1$. In fact, $\phi=\phi(r)$.

For notational convenience, in the following we denote $B(0, R)$ by $B$. Using equation (3.2) we have

$$
\begin{aligned}
S^{\prime}(t)= & \frac{1}{\sigma+1} \int_{B} p^{\sigma+1} \Delta \phi-\frac{1}{\sigma+1} \int_{\partial B} p^{\sigma+1} \frac{\partial \phi}{\partial n} \\
& -m \int_{B}(\nabla p \cdot x) \phi-\frac{1}{\beta-1} \int_{B} p \phi+\int_{B} p^{\beta} \phi \\
= & -\frac{\lambda_{R}}{\sigma+1} \int_{B} p^{\sigma+1} \phi-m \int_{B}(\nabla p \cdot x) \phi \\
& -\frac{1}{\beta-1} \int_{B} p \phi+\int_{B} p^{\beta} \phi .
\end{aligned}
$$

Since $p_{r} \leq 0$, we obtain

$$
\int_{B} \nabla p \cdot x \phi d x=\int_{S^{N-1}} d \sigma \int_{0}^{R} p_{r}(r, t) \phi(r) r^{N} d r \leq 0
$$

and

$$
\begin{aligned}
\int_{B} p^{\sigma+1} \phi & =\int_{\{p \geq 1\} \cap B} p^{\sigma+1} \phi+\int_{\{p<1\} \cap B} p^{\sigma+1} \phi \\
& \leq \int_{\{p \geq 1\} \cap B} p^{\beta} \phi+\int_{\{p<1\} \cap B} p \phi \leq \int_{B} p^{\beta} \phi+\int_{B} p \phi .
\end{aligned}
$$

Hence

$$
\begin{aligned}
S^{\prime}(t) & \geq\left(1-\frac{\lambda_{R}}{\sigma+1}\right) \int_{B} p^{\beta} \phi-\left(\frac{\lambda_{R}}{\sigma+1}+\frac{1}{\beta-1}\right) \int_{B} p \phi \\
& \geq\left(1-\frac{\lambda_{R}}{\sigma+1}\right) S^{\beta}(t)-\left(\frac{\lambda_{R}}{\sigma+1}+\frac{1}{\beta-1}\right) S(t)
\end{aligned}
$$

by Jensen's inequality. 
On the other hand,

$$
\begin{aligned}
& \left(1-\frac{\lambda_{R}}{\sigma+1}\right) S^{\beta}\left(t_{0}\right)-\left(\frac{\lambda_{R}}{\sigma+1}+\frac{1}{\beta-1}\right) S\left(t_{0}\right) \\
& =\left(1-\frac{\lambda_{R}}{\sigma+1}\right) Q^{\beta}-\left(\frac{\lambda_{R}}{\sigma+1}+\frac{1}{\beta-1}\right) Q>0,
\end{aligned}
$$

by the condition in the claim. Integrating (3.5) we obtain that $S(t)$ blows up in finite time. Therefore $p(x, t)$ blows up in finite time and the proof is thus completed.

It will be instructive to compare our result with the analysis for $N=1$. As mentioned in the introduction, in that case the method of intersection comparison is applied on exact self-similar solutions with the same blow-up time. So the spatial structure of an unbounded solution close to the final blow-up time could be analyzed quite precisely. Hence there hold some important estimates on the size of the support of unbounded solutions. For example, one has the following estimate:

$$
\text { meas } u\left(T^{-}\right) \leq \text {meas } u_{0}+C T^{m},
$$

where $C>0$ is a constant which depends only on $\sigma, \beta$.

It seems hard to get such a precise estimate in high dimensions, for one does not have Sturmian type comparison. But as we will see below, if $\beta>\sigma+3$, one can get a similar estimate. It is quite reasonable to believe that it should be true for all $\beta>\sigma+1$. Unfortunately we have not found a proof yet, since the argument strongly relies on Proposition 2.3.

Proposition 3.4. If $\beta>\sigma+3$, then

$$
\operatorname{supp} u\left(\cdot, T^{-}\right) \subset B\left(0, K^{\prime}\right),
$$

where $K^{\prime}=C_{0} R_{0}+C_{1} T^{m}, C_{i}, i=0,1$, are positive constants independent of $R_{0}, T$.

Proof. Recall from Proposition 2.3 that

$$
u_{2}\left(x_{1}, t\right)=(1+t)^{-\frac{1}{\beta-1}} \psi\left(\xi_{1}\right), \xi_{1}=\frac{x_{1}}{(1+t)^{m}},
$$

satisfies

$$
\left(u_{2}\right)_{t} \geq\left(\left(u_{2}\right)^{\sigma}\left(u_{2}\right)^{\prime}\right)^{\prime}+\left(u_{2}\right)^{\beta+1},
$$

where $\psi\left(\xi_{1}\right)=A\left(1-\frac{\xi_{1}^{2}}{a^{2}}\right)_{+}^{\frac{1}{\sigma}}$.

Since the hypothesis of Lemma 3.3 holds, we can choose $R_{3}>R_{0}$ so that $u(x, t) \leq$ $(T+1)^{-\frac{1}{\beta-1}} A$ for all $|x| \geq R_{3}, t>0$.

Set $w_{2}(x, t) \equiv u_{2}\left(x_{1}-R_{3}, t\right)$.

Notice that $w_{2}(x, t)=(t+1)^{-\frac{1}{\beta-1}} A>(T+1)^{-\frac{1}{\beta-1}} A \geq u(x, t)$ on $\left\{x \mid x_{1}=\right.$ $\left.R_{3}\right\} \times[0, T), w_{2}(x, 0) \geq u(x, 0)$ if $x_{1} \geq R_{3}$. Since

$$
\left(w_{2}\right)_{t} \geq \nabla\left(w_{2}^{\sigma} \nabla w_{2}\right)+w_{2}^{\beta+1},
$$

by comparison again, it leads to $w_{2}(x, t) \geq u(x, t)$ on $\left\{x \mid x_{1} \geq R_{3}\right\} \times[0, T)$. So

$$
\operatorname{supp} u\left(\cdot, T^{-}\right) \subset\left\{x \mid x_{1} \leq R_{3}+a(T+1)^{m}\right\} .
$$

As before, one concludes

$$
\operatorname{supp} u\left(\cdot, T^{-}\right) \subset B\left(0, R_{3}+a(T+1)^{m}\right) .
$$


We note that as in the proof of Lemma 3.3 , the number $R_{3}>R_{1}$ only needs to satisfy

$$
R_{3}^{-\frac{2}{\beta-(\sigma+1)}} \leq C(T+1)^{-\frac{1}{\beta-1}} A,
$$

while $R_{1}$ has the form $R_{1}=2 R_{0}+R T^{m}$ for some large $R$ independent of $R_{0}$ and $T$, as in (3.3). So we can choose $R_{3}=C R_{0}+C T^{m}$. Hence the proposition is proved.

Note that for fixed $R_{0}$, if $T \gg 1$, the estimate above is better than what we obtained in the proof of Lemma 3.3. The proposition above also provides another proof for Theorem 1.1 in the case $\beta>\sigma+3$.

\section{Effective Localization in a Special Case}

The proof is pretty much the same as for Theorem 1.1. Hence in the following we just give a sketch.

Sketch of the proof. Since $u_{0}$ is not necessarily compactly supported, the Alexandroff's reflection principle (Lemma 3.1) does not hold automatically. But the extra symmetry assumption recovers the lemma.

Observe that by uniqueness, $u(x, t)$ has the same symmetry as $u_{0}$. Take the derivative of equation (1.1) with respect to $r$, and then notice that $r^{N-1} u_{r}$ satisfies a good linear PDE. Using the comparison principle on the half-line we have $u_{r} \leq 0$ for $r \geq 0, t>0$. But that means the solution is radial nonincreasing. By replacing $2 R_{0}$ with any positive number, the conclusion of Lemma 3.1 is trivially true.

Hence one can find an $R$ such that

$$
u(x, t)<\theta_{0}(T-t)^{-\frac{1}{\beta-1}} \text {, for }|x|>R, t>0 .
$$

Notice that as in Theorem 1.1, the proof for this fact only depends on the validity of the conclusion of Lemma 3.1.

Following the argument in Lemma 3.2, by asymptotic behavior of $u_{0}$, there exists $R_{1}>0$ such that $u(x, 0) \leq w(x, 0)$ if $x_{1} \geq R_{1}$, where $w(x, t)=u_{s}\left(x_{1}-R_{1}, t\right), u_{s}$ is defined in section 2 .

So the same comparison arguments imply immediately that

$$
\sup _{0<t<T} u(x, t) \rightarrow 0, \text { as }|x| \rightarrow+\infty .
$$

In particular, it implies that the blow-up set is compact.

Remark 1 . We would like to compare Theorem 1.2 with the following result, which is essentially contained in [19] (see also [13] and 9]).

Proposition 4.1. If $N>2,0 \leq u_{0} \leq L|x|^{-\frac{2}{\beta-(\sigma+1)}}$ in $R^{N}$, and $\frac{N}{N-2}<\frac{\beta}{\sigma+1}<p_{c}$, then $u$ exists globally in time (hence the blow-up set is empty).

The function $L|x|^{-\frac{2}{\beta-(\sigma+1)}}$ is a singular solution, which satisfies the equation pointwise except at the origin, where $L, p_{c}$ are constants depending on $N, \sigma, \beta$ only. Notice that in this case $\beta>\sigma+1+\frac{2}{N}$ holds automatically.

Note that equation (1.1) and its semilinear counterpart (i.e, $\sigma=0$ ) have similar stationary solutions up to a suitable change of variables. So the argument in [19] (see also [12] for further studies) can be modified to prove Proposition 4.1. 
Theorem 1.2 can be viewed, in some sense, as an extension of the above result under extra symmetry assumptions. However, it seems hard to obtain these properties in the quasilinear situation by the methods in [19] and [12].

Remark 2. In the proof above the symmetry condition is only required to ensure the Alexandroff's reflection principle. It will be interesting to find more general initial data to make some kind of comparison preserved for all $t>0$. If so, with the same asymptotic assumption, we can conclude the effective localization. It is conjectured in [16 that the effective localization should be true if we only assume that $u_{0}$ decays to zero at infinity, which is still open. Related results for radial cases can be found in [8], where the Friedman-McLeod method was applied to general quasilinear parabolic equations under more restrictive conditions. The similar method is also used to show that single point blow-up could occur for Cauchy problem with compactly supported initial data in [2]. On the other hand, intersection comparison technique was used in [6] to deal with initial data with special decay at infinity. Our radial symmetry case could probably be studied by the intersection comparison technique or the Friedman-McLeod method. We note that the method used here would be simpler.

\section{ACKNOWLEDGEMENT}

The first author was partially supported by NSF grant DMS-0140604. The authors would like to thank Prof. Galaktionov for helpful correspondence.

\section{REFERENCES}

[1] D. Aronson, L. Caffarelli, The initial trace of the solution of the porous medium equation, Trans. Amer. Math. Soc 280 (1983), 351-366. MR 85c:35042

[2] F. V. Bunkin, V. A. Galaktionov, N. A. Kirichenko, S. P. Kurdyumov and A. A. Samarski, A nonlinear boundary value problem of ignition by radiation. Zh. Vychisl. Mat. i Mat. Fiz. 28 (1988), no. 4, 549-559, 623; English transl. in U.S.S.R. Comput. Math. and Math. Phys. 28 (1988), no. 2, 157-164 (1989). MR 89d:80008

[3] C. Cortazar, M. Del Pino and M. Flgueta, On the blow-up set for $u_{t}=\Delta u^{m}+u^{m}, m>1$, Indiana Univ. Math. J. 47 (1998), 541-561. MR 99h:35085

[4] A. Friedman, B. McLeod, Blow-up of positive solutions of semilinear heat equations, Indiana Univ. Math. J. 34 (1985), 427-447. MR 86j:35089

[5] V. A. Galaktionov, Proof of the localization of unbounded solutions of the nonlinear parabolic equation $u_{t}=\left(u^{\sigma} u_{x}\right)_{x}+u^{\beta}$, Differ. Equations 21 (1985), 15-23. MR 86d:35068

[6] V. A. Galaktionov, Asymptotic behavior of unbounded solutions of the nonlinear equation $u_{t}=\left(u^{\sigma} u_{x}\right)_{x}+u^{\beta}$ near a "singular" point. Soviet Math. Dokl., 33 (1986), 840-844. MR $87 \mathrm{j}: 35052$

[7] V. A. Galaktionov and J. Vazquez, The problem of blow-up in nonlinear parabolic equations. Current developments in partial differential equations (Temuco, 1999). Discrete Contin. Dyn. Syst. 8 (2002), no. 2, 399-433. MR 2003c:35067

[8] V. A Galaktionov and J. Vazquez, Blow-up for quasilinear heat equations described by means of nonlinear Hamilton-Jacobi equations. J. Differential Equations 127 (1996), 1-40. MR 97e:35080

[9] V. A Galaktionov and J. Vazquez, Continuation of blowup solutions of nonlinear heat equations in several space dimensions. Comm. Pure Appl. Math. 50 (1997), 1-67. MR 97h:35085

[10] Y. Giga, R. Kohn, Asymptotically self-similar blow-up of semilinear heat equations, Comm. Pure Appl. Math. 38 (1985), 297-319. MR 86k:35065

[11] C. Gui, Symmetry of the blow-up set of a porous medium equation, Comm. Pure Appl. Math. 48 (1995), 471-500. MR 96e:35087

[12] C. Gui, W. Ni and X. Wang, Further study on a nonlinear heat equation, J. Diff. Equat. 169 (2001), 588-613. MR 2002b:35088 
[13] C. Gui, W. Ni and X. Wang, On the stability and instability of positive steady states of a semilinear heat equations in $R^{n}$, Comm. Pure Appl. Math. 45 (1992), 1153-1181. MR 93h:35095

[14] A. S. Kalashnikov, Some problems of the qualitative theory of non-linear degenerate secondorder parabolic equations, Russian Math. Surveys 42 (1987), 169-222. MR 88h:35054

[15] H. Levine, The role of critical exponents in blow-up problems, SIAM Review 32 (1990), 262-288. MR 91j:35135

[16] A. Samarskii, V. Galaktionov, V. Kurdyumov and A. Mikhailov, Blow-up in quasilinear parabolic equations, Nauka, Moscow, 1987; English translation: Walter de Gruyter, Berlin/New York, 1995. MR 96b:35003

[17] J. L. Vazquez, An introduciton to the mathematical theory of the porous medium equation, Shape Optimization and Free Boundaries, M. C. Delfour ed., Mathematical and Physical Sciences, Series C, vol 380, Kluwer Ac. Publ. Boston and Leiden; 1992. MR 94h:49005

[18] J. Velazquez, Estimates on $(N-1)$-dimensional Hausdorff measure of the blow-up set for a semilinear heat equation, Indiana Univ. Math. J. 42 (1993), 445-476. MR 94g:35031

[19] X. Wang, On the Cauchy problem for reaction-diffusion equations, Trans. Amer. Math. Soc. 337 (1993), 549-589. MR 93h:35106

[20] F. Weissler, Single point blow-up for a semilinear initial value problem, J. Differ. Equat. 55 (1984), 204-224. MR 86a:35076

Department of Mathematics, University of Connecticut, Storrs, Connecticut 06269

E-mail address: gui@math.uconn.edu

The Fields institute, 222 College Street, Toronto, Ontario, Canada M5T 3J1

E-mail address: xkang@fields.utoronto.ca 